\title{
An Efficient Bi-Level Surrogate Approach for Optimizing Shock Control Bumps under Uncertainty
}

\author{
Christian Sabater* and Stefan Görtz ${ }^{\dagger}$ \\ German Aerospace Center (DLR), Institute of Aerodynamics and Flow Technology, 38108 Braunschweig, Germany
}

The assessment of uncertainties is essential in aerodynamic shape optimization problems in order to come up with configurations that are more robust. The influence of aleatory fluctuations in flight conditions and manufacturing tolerances is of primary concern when designing shock control bumps, as their effectiveness is highly sensitive to the shock wave location. However, exploring the stochastic design space for the global robust optimum increases the computational cost, especially when dealing with nonconvex design spaces and multiple local optima. The aim of this paper is to develop a framework for efficient aerodynamic shape optimization under uncertainty by means of a bi-level surrogate approach and to apply it to the robust design of a retrofitted shock control bump over an airfoil. The framework combines a surrogate-based optimization algorithm with an efficient surrogate-based approach for uncertainty quantification. The surrogate-based optimizer efficiently finds the global optimum of a given quantile of the drag coefficient. It outperforms traditional evolutionary algorithms by effectively balancing exploration and exploitation through the combination of adaptive sampling and a moving trust region. At each iteration of the optimization, the surrogate-based uncertainty quantification uses an active infill criterion in order to accurately quantify the quantile of the drag at a reduced number of function evaluations. Two different quantiles of the drag are chosen, the $95 \%$ to increase the robustness at off-design conditions, and the $50 \%$ for a configuration that is best for day to day operations. In both cases, the optimum configurations lead to an airfoil that is more robust to geometrical and operational uncertainties, compared to the configuration obtained through classical deterministic optimization.

\section{Nomenclature}

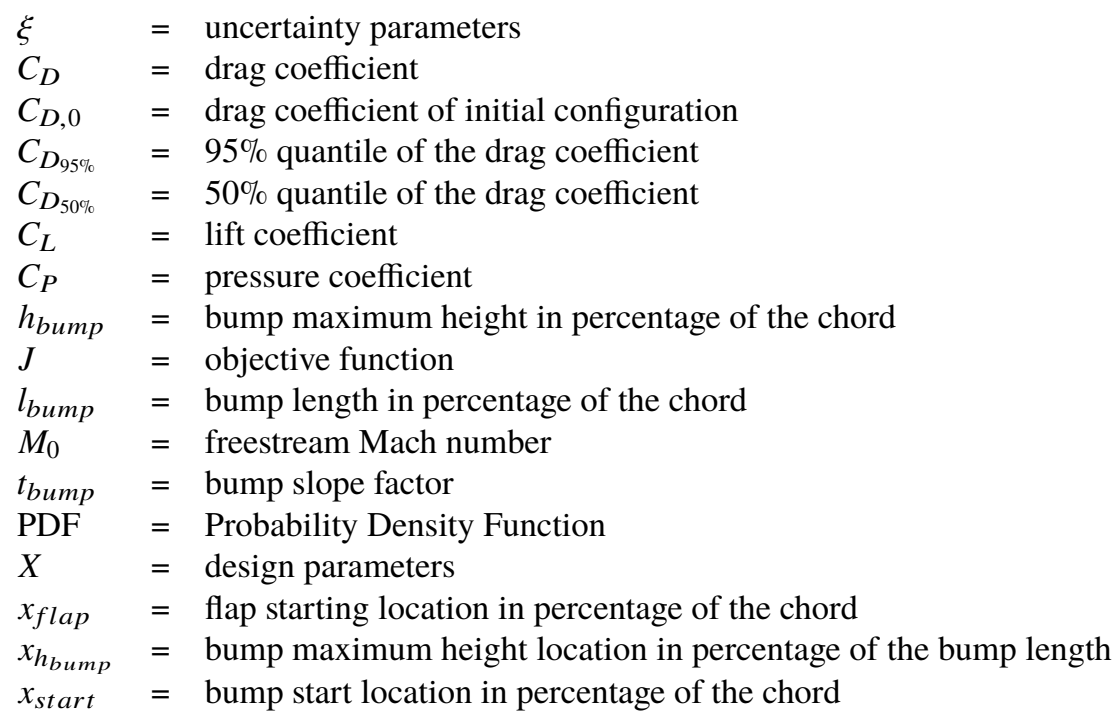

\footnotetext{
*PhD student, $\mathrm{C}^{2} \mathrm{~A}^{2} \mathrm{~S}^{2} \mathrm{E}$, Lilienthalplatz 7, AIAA Member

$\dagger$ Team Leader, $\mathrm{C}^{2} \mathrm{~A}^{2} \mathrm{~S}^{2} \mathrm{E}$, Lilienthalplatz 7, AIAA Member
} 


\section{Introduction}

Shock control bumps (SCBs) are passive flow control devices that improve the performance of transonic wings by altering the flow around near-normal shock waves. It is the most effective device to reduce wave drag when applied to flow with strong stationary shock waves [1]. Shock control bumps split the shock into weaker shocks by means of oblique or compression waves [2]. The flow is isentropically (gradually) decelerated with respect to the baseline configuration where no bump is present. For turbulent airfoils at high lift coefficients or high Mach numbers, when the shock wave strength is increased, the performance of SCBs is improved as they can further reduce wave drag.

Shock Control bumps were first introduced by Ashill in 1992 for the mitigation of wave drag [3]. Further studies took place in Europe within the EUROSHOCK II project [1] and in the USA [4] to investigate its full potential. From the 2000s, the focus has been on understanding the flow physics [5] and the realization of optimization studies [6], [7]. A crucial aspect that is gaining more attention recently is the need for a robust SCB configuration for industrial applications [8], [9]. A complete overview of Shock Control Bumps is given by Bruce and Collis [10].

The robustness of shock control bumps, i.e., its ability to effectively reduce wave drag at different flight conditions, is of primary concern as they are highly sensitive to the shock wave location. At freestream velocities or lift coefficients different from their design point, SCBs suffer from adverse effects as the shock wave is not located in the designed location [2]. For example, when the shock wave moves upstream (due to a decrease in flight speed or lift coefficient), the flow is re-accelerated due to the curvature of the bump, leading to a second supersonic region behind the first shock. On the contrary, if the shock is located downstream of the SCB, no lambda shock structure is generated and the flow is further accelerated, leading to an increased shock strength and possibly causing flow separation.

A state of the art solution to deal with different freestream velocities and lift coefficients is the use of an adaptive shock control bump that can be deployed according to the known flight conditions [11]. Previous studies showed that the optimum bump height is directly proportional to the shock strength [3]. The optimum bump height can be determined for each flight condition. In addition, by means of variable chamber it is possible to shift the shock wave location to the one that is ideal in terms of shock strength reduction by the SCB. The adaptive bump can be optimized following a classical multi-point approach following operational requirements, as shown in Fig. 11. However, this approach does not take into account day to day small aleatory fluctuations in flight conditions (shaded area in Fig 1). Thus, the traditional deterministic optimization setup for the design of shock control bumps, be it single-point or multi-point, is not an accurate representation of the reality, leading to SCBs that do not take into account operational and geometrical uncertainties.
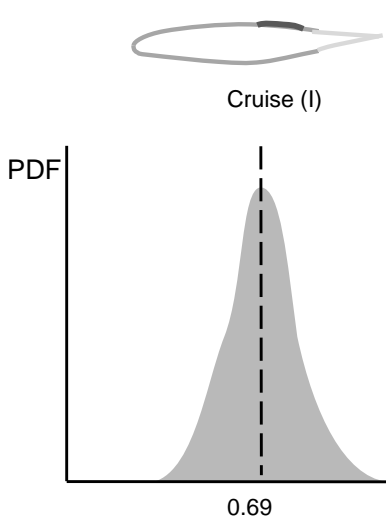

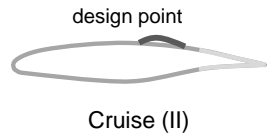

Cruise (II)

Fig. 1 Multi-point design at different (known) freestream Mach numbers (dashed lines). At each flight condition irreducible uncertainties are present

The design point of an aircraft (cruise lift coefficient at a given Mach number) is used by engineers to simplify the design process. Even a multi-point representation does not fully represent reality as shown in Fig. 11. In reality, stochastic operating conditions as well as manufacturing tolerances affect the real performance of SCBs. These need to be taken into account in the design process. As a result, it is necessary to directly assess the effect of relatively small unknown, aleatory (or irreducible) uncertainties.

Robust optimization techniques can cope with the realistic design of SCBs by accounting for the uncertainties during the design stage. Therefore they prevent a configuration that does not match the specifications for which it was designed 
when slightly deviating from the design conditions [12]. However, when uncertainty is added to global optimization algorithms, the computational cost may increase by several orders of magnitude. The objective function is changed from a deterministic quantity of interest, i.e. drag coefficient at a given flight condition, to a statistic of the quantity of interest. As a result, the stochastic space needs to be characterized at each iteration of the optimization [13]. This can be unfeasible if the underlying model is expensive to evaluate such as the case of simulations involving Computational Fluid Dynamics (CFD). To deal with this problem, it is necessary to reduce both the number of optimization iterations and the number of evaluations required for the uncertainty quantification (UQ).

In this paper, an efficient framework for the Optimization Under Uncertainty for the aerodynamic shape optimization of shock control bumps is presented. The global optimizer follows a modified surrogate-based optimization (SBO) [14] approach that is designed to carefully balance exploration and exploitation. At each iteration of the optimization, a Surrogate-Based Uncertainty Quantification (SBUQ) approach is used to efficiently obtain a given $\tau$ quantile of the drag coefficient. The optimization of a given quantile is more flexible than other classical approaches for robust optimization such as the expectation. According to the design requirements, medium or high quantiles can be chosen as objective function. The trade-off in this selection will be analyzed for two different robust optimum configurations, and compared to the deterministic optimum configuration.

\section{Problem Definition}

This section introduces the parametrization of the design parameters and uncertainties, the formulation of the deterministic and robust optimization, the numerical model and the CFD process chain. The classical deterministic problem setup serves as verification of the optimization process at a single flight condition and will be compared with the robust solution.

Both the deterministic and the robust formulations aim to find the optimum bump shape to be retrofitted to the current supercritical RAE 2822 airfoil for the operation at dash condition: Mach number of 0.734 and constant lift coefficient of 0.789 (angle of attack of 2.77 degrees), which is defined by a higher freestream velocity than the design point. At this condition a strong near-normal shock wave is present over the upper surface and the addition of a shock control bump is expected to considerably reduce drag by weakening the shock intensity. Previous studies have been done for the aerodynamic shape optimization of airfoils under uncertainty [15, 16]. However, no focus was given in obtaining a robust global solution that reduces both the number of optimization iterations and function evaluations in the uncertainty quantification stage. In addition, the current robust design optimization aims to considerably improve the performance of the airfoil by modifying only the shape in the region near the shock wave.

\section{A. Parametrization of the Design Vector}

The bump is defined as a local perturbation to the original airfoil by means of a Hicks-Henne Sinusoidal Function with 5 design parameters, following the parametrization of Mazaheri [17] and Tian [18]. The slope of the curvature of this function is zero at both ends. As a result, no discontinuities are present between the bump and the airfoil. A second advantage with respect to other parametrization such as NURBS curves is that the Hicks-Henne parameters represent the physical geometry of the bump, allowing for a straightforward definition of the design space as shown in Table 1 . The parameters are normalized according to the airfoil chord length, $c$.

$$
z(x)=h_{\text {bump }}\left[\sin \left(\pi\left(\frac{x-x_{\text {start }}}{l_{\text {bump }}}\right)^{m}\right)\right]^{t_{\text {bump }}}, \quad x_{\text {start }} \leq x \leq x_{\text {start }}+l_{\text {bump }}
$$

where $m$ is used to modify the asymmetry of the bump:

$$
m=\frac{\log (0.5)}{\log \left(x_{h_{\text {bump }}}\right)}
$$

The bounds of the parameters have been chosen according to physical constraints in order to reach a realistic design. For example, a bump height of 0 is equivalent to the baseline configuration of an airfoil with no bump. The upper limit of $0.015 c$ is chosen having into account that the order of magnitude of the bump height is strongly related to the boundary layer displacement thickness. The location of the bump is centered along the shock wave location of the initial airfoil, around 55\% of the chord. The design parameters $X$ are normalized between 0 and 1, equivalent to the lower and upper bounds of Table 1 


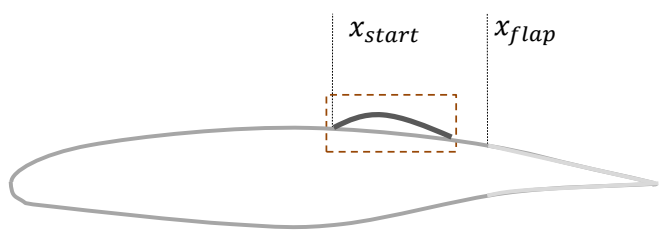

a)

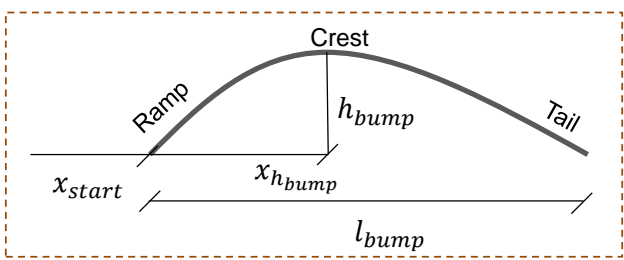

b)

Fig. 2 Parametrization of the Shock Control Bump: a) in relation with the airfoil; b) zoom in of the bump and design parameters

Table 1 Design parameters of the shock control bump

\begin{tabular}{c|l|c|c} 
Parameter & Description & Lower Bound & Upper Bound \\
\hline$h_{\text {bump }} / c$ & Bump maximum height & 0 & 0.015 \\
$x_{h_{\text {bump }}} / c$ & Bump maximum height location (as a function of the bump length) & 0.4 & 0.85 \\
$l_{\text {bump }} / c$ & Bump length & 0.15 & 0.45 \\
$x_{\text {start }} / c$ & Bump starting location & 0.3 & 0.54 \\
$t_{\text {bump }}$ & Bump slope factor & 0.2 & 2
\end{tabular}

\section{B. Parametrization of Uncertainties}

Table 2 shows the operational and geometrical uncertainties chosen for this problem. Mach number and lift coefficient strongly affect the shock wave location, while the SCB height is the most important geometrical parameter influencing the smearing effect of the shock wave [3].

Each uncertainty follows a beta distribution with the mean $\mu_{\xi}$, according to nominarl conditions, and standard deviation $\sigma_{\xi}$ according to Table 2 A main advantage of beta distributions for UQ is that they are bounded (truncated PDF). They represent a more realistic representation of the uncertainties than unbounded normal distributions. Also, beta distributions are able to adapt to both normal and uniform distributions by modifying their shape parameters $\alpha_{1}$ and $\alpha_{2}$. In this case the distribution is required to be symmetric $\left(\alpha_{1}=\alpha_{2}=5\right)$ to resemble a truncated normal distribution. Then, the location $\beta_{1}$ and scale $\beta_{2}$ are calculated to have the required mean $\mu$ and standard deviation $\sigma$.

\section{Table 2 Uncertainties in the optimization under uncertainty problem}

\begin{tabular}{|c|c|c|c|}
\hline Parameter & Description & Mean & Standard Deviation \\
\hline$M_{0, \xi}$ & Freestream Mach number uncertainty & 0.734 & 0.0045 \\
\hline$C_{L_{0, \xi}}$ & Lift coefficient uncertainty & 0.791 & 0.0045 \\
\hline$h_{\text {bump }, \xi} / c$ & Bump height uncertainty & 0 & 0.008 \\
\hline \\
\hline
\end{tabular}

\section{Deterministic Optimization Problem}

The deterministic global solution follows from the classical drag optimization at constant lift coefficient, $C_{L}=0.789$, and freestream Mach number, $M_{0}=0.734$ :

$$
J^{*}\left(X^{*}\right)=\min _{X} \frac{C_{D}\left(X, M_{0}, C_{L}\right)}{C_{D_{0}}}
$$

Under the constraint:

$$
c_{1}: x_{\text {start }}+l_{\text {bump }}-x_{\text {flap }} \leq 0
$$


The drag coefficient depends on the design variables $X$ that modify the retrofitted bump. The constant lift coefficient is handled internally by the CFD solver as it iteratively changes the angle of attack during the solution. The drag is normalized by the initial drag of the baseline airfoil without bump, $C_{D_{0}}$. For example, a value equal or greater than 1 of the objective function means that the bump deteriorates the performance of the clean wing.

The constraint guarantees that the end of the bump does not interfere with the flap (last $20 \%$ of the chord). As shown in subsection II.A by defining the bump starting location $x_{\text {start }}$ and length $l_{\text {bump }}$, it is not possible to fix the its end through the bounds of these parameters. The constraint is introduced by a quadratic penalty function within the optimization in the surrogate. This approach allows the construction of a continuous surrogate in the design space (without penalty function), while avoiding the exploration of locations where the penalty is active.

\section{Robust Optimization Problem}

The robust solution involves both operational and geometric uncertainties by taking into account the realistic stochastic conditions in flight. As a result, the drag coefficient (objective function) becomes a random variable. A reliability-based approach is followed by minimizing a given $\tau$ quantile of the drag. Two different cases are considered: a high quantile, $\tau=95 \%$, and the median, $\tau=50 \%$.

$$
J(X, \xi)=\min _{X, \xi} \frac{C_{D_{\tau} \sigma_{0}}(X, \xi)}{C_{D_{0}}}
$$

The optimization of the $95 \%$ quantile, $C_{D, 95 \%}$, is equivalent to the minimization of the value below which $95 \%$ of the smallest realizations of drag occur due to the uncertainties. By properly accounting for the uncertainties in the design process, confidence intervals can be used instead of conservative margins of safety. On the other hand, by optimizing the $50 \%$ quantile, $C_{D, 50 \%}$, the focus is in the middle value of the realizations, without an interest in extreme events. It is expected that two different configurations will arise. The designer will be responsible to come up with the best trade-off by selecting the proper quantile.

\section{E. Numerical Model}

In order to obtain the aerodynamic performance of the airfoil, the Reynolds Average Navier Stokes (RANS) equations are solved by means of the DLR TAU solver [19] with the negative Spalart-Allmaras turbulent model. In particular it is used a $3 \mathrm{v}$ multigrid circle, the lower/upper symmetric Gauss-Seidel implicit method for time integration in backward Euler solver and a central flux discretization. A converged density residual of $1 \mathrm{e}-8$ is chosen. The unstructured mesh is hybrid and quasi two-dimensional, with tetrahedral and prism elements, as shown in Fig. 3 . The number of grid nodes is 29,000 , with 380 surface nodes around the airfoil. The domain is decomposed in four regions following the TAU built-in partitioner for the efficient parallelization of the solution.

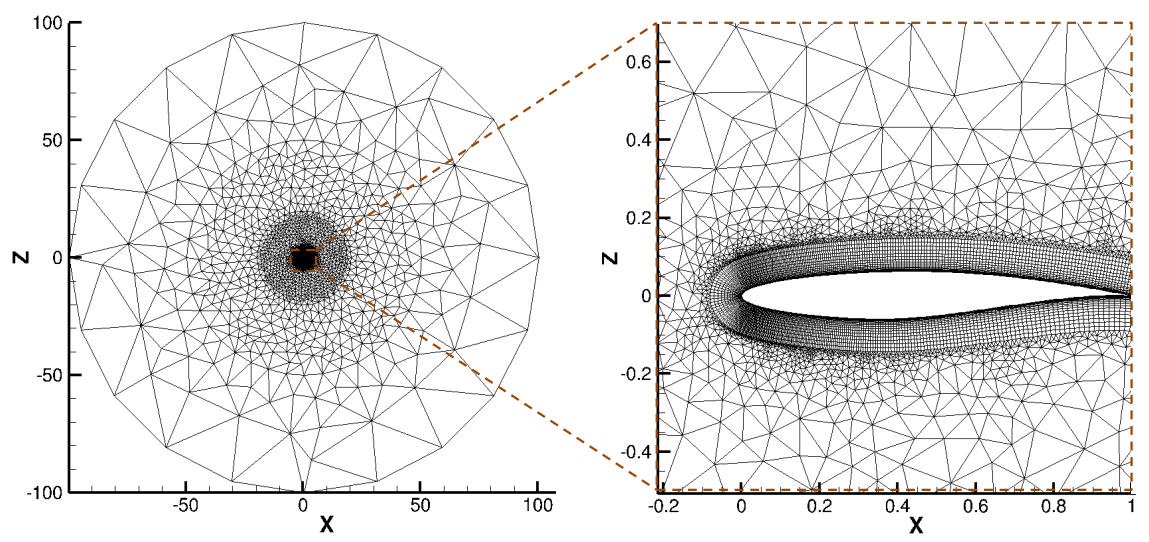

Fig. 3 CFD Grid used in the Optimization Problem 


\section{F. Process Chain}

Every time the drag coefficient is required from a given design vector $X_{i}$, (for deterministic optimization), or from a given design vector $X_{i}$ and uncertainties $\xi$, (for the UQ in robust design), the process chain of Fig. 4 is active. The geometry is changed by means of a mesh deformation tool employing radial basis functions developed by DLR [20]. After the addition of the boundary conditions, the RANS equations are solved. The drag coefficient, $C_{D}$, is obtained by the integration of the pressure and viscous forces along the surface of the airfoil. The process chain is controlled by Flow Simulator [21] from Python and is able to handle convergence errors, restarts and parallelization.

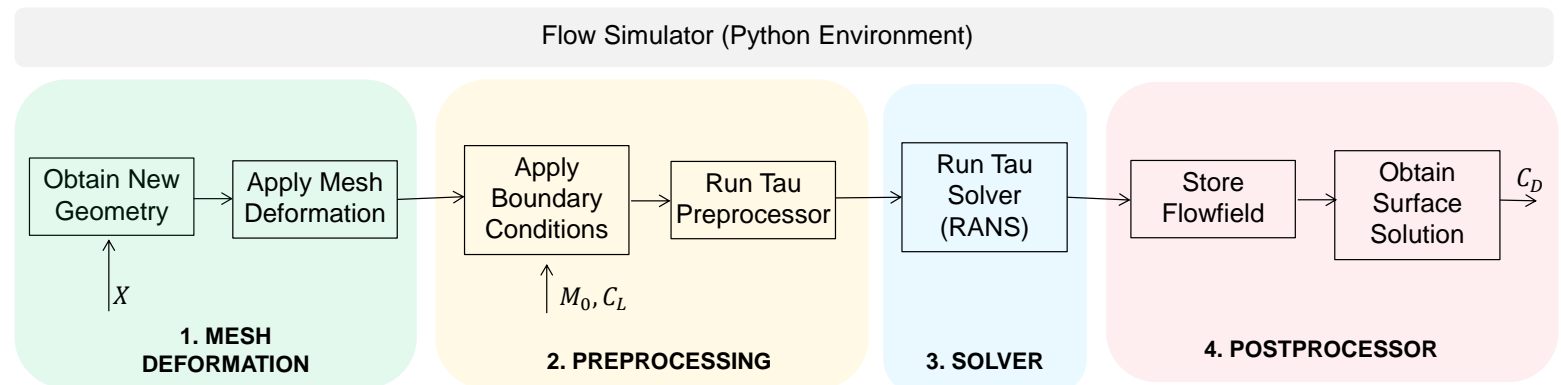

Fig. 4 Framework for the evaluation of the objective function through CFD, from the design vector and operating conditions to the drag coefficient

\section{Optimization Under Uncertainty Framework}

Global optimization problems require a large number of function evaluations to find the global minimum. This can be unfeasible if the underlying model is expensive to evaluate such as the case of simulations involving Computational Fluid Dynamics. In the case of optimization under uncertainty, the computational cost is several orders of magnitude more expensive, as a characterization of the stochastic space should be done at each iteration. To overcome this problem, a framework for the efficient optimization under uncertainty applied to expensive black box problems is presented below. First the outer layer (surrogate-based optimization) and inner layer (surrogate-based uncertainty quantification) are introduced, followed by its combination in the optimization under uncertainty approach.

\section{A. Surrogate-Based Optimization}

The aim of surrogate-based optimization is to make the optimization more efficient by reducing the number of function evaluations of the expensive CFD solver [22]. A surrogate model that is cheaply evaluated is built following the parametrization and the quantity of interest. The Surrogate-Modelling for Aero-Data Toolbox (SMARTy) developed by DLR is used for the initial Design of Experiments sampling and for the creation of the Kriging surrogate [23].

The overall framework of the optimization process is shown in Fig. 5. In case of deterministic optimization, the objective function is the drag coefficient obtained with the process chain introduced in Fig. 4. When dealing with optimization under uncertainty, the objective function is the quantile of the drag that will be obtained through the uncertainty quantification process later introduced in Fig. 6. The framework has been tailored for the efficient application to aerodynamic shape optimization problems. Each of the steps in the optimization process is explained below.

\section{Design of Experiments}

One of the key elements for the construction of surrogate models is the sampling strategy for the evaluation of the objective function. The Design of Experiments (DoE) aims to analyze the data efficiently by computing better experiments. The most common sampling approach is the use of Latin Hypercube Sampling developed by Mckay [24]. However, Quasi Monte Carlo techniques employing deterministic low discrepancy sequences [25] can prove more effective to obtain an evenly sampling distribution with a very good space filling capability [26]. As a result it can better represent the design space with few sample points.

Sobol Sequences are a quasi-random low discrepancy sequence that use a base of two to successively create fined uniform partitions of the unit interval [27]. In contrast with Latin Hypercube Square, Sobol sampling is deterministic. 


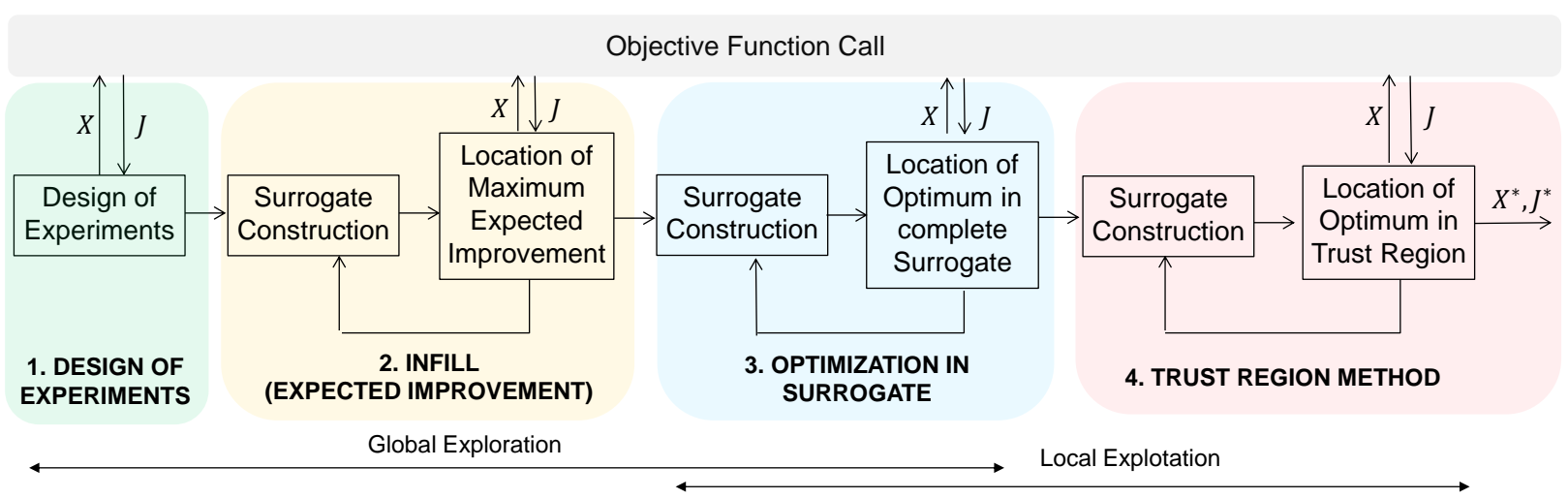

Fig. 5 Surrogate-based optimization framework to efficiently find global minimum of complex functions

The addition of more samples is straightforward by continuing the sequence without degradation in the space filling. As a result, it is possible to improve its accuracy as more samples are added by reusing the existing points.

When approximating the objective function with a Gaussian process, the number of samples for an effective initial design of experiments should be about ten times the input dimension [28]. As a result, an initial sampling strategy of 50 samples based on Sobol sequences is chosen for the five dimensional SCB optimization problem.

\section{Surrogate Construction}

Once the initial sampling is evaluated, the surrogate model is constructed. The approximation of the objective function by Kriging, using Bayesian statistics, is among the most used surrogate models in optimization [22, 29]. A detailed explanation of the construction of Kriging surrogates is given by Forrester in [30].

In particular, Universal Kriging with a Gaussian Kernel (exponent fixed to 2) is used. The hyperparameters of the correlation model are fine-tuned by the maximization of the expected improvement [31]. In order to obtain a global solution, this optimization is carried out with differential evolution [32]. A regularization constant, also called nugget effect, is added to convert the interpolation into regression in order to better fit noisy data.

\section{Refinement}

The Kriging surrogate provides the normal distribution prediction of the objective function at any given point (mean $\hat{y}$ and standard deviation $\hat{\sigma}(X)$ ). This makes possible the calculation of the probability of improvement from the best sampled solution $y_{\min }$. The Expected Improvement (EI) is then defined as the product of the improvement between the potential better solution, $\hat{y}(X)$, and the current best one, $y_{\min }$ [14].

$$
E[I(X)]=\left(y_{\min }-\hat{y}(X)\right) \Phi\left(\frac{y_{\min }-\hat{y}(X)}{\hat{\sigma}(X)}\right)+\hat{\sigma}(X) \phi\left(\frac{y_{\min }-\hat{y}(X)}{\hat{\sigma}(X)}\right)
$$

where $\Phi$ and $\phi$ are respectively the cumulative and probability distribution functions of the standard normal distribution. The EI balances exploration with exploitation. A large expected improvement is present in regions where a smaller solution than the current best is possible (first term of equation 7 ) and/or when the model error is large (second term of equation 7 .

During the refinement phase, the location with maximum EI on the Kriging model is obtained by differential evolution and used as infill criteria to update the surrogate model. This process is repeated iteratively until a given tolerance is reached, or until the number of function evaluations reserved for this stage is reached.

The EI criteria can be used in many cases as the best route to find the global minimum. However, in other occasions it may provide a very slow convergence rate [22]. As a result, two further stages are introduced to guarantee convergence.

\section{Iterative Optimization in Surrogate}

Once the refinement is finished, the optimization is performed within the surrogate through differential evolution. A population size ten times the number of design parameters is selected. By selecting a conservative convergence criteria, it is possible to obtain the global minimum of the surrogate at relatively low cost. The optimum point in the surrogate is 
validated and computed in the CFD. It is unlikely that both surrogate and CFD solution will match, so the surrogate is recomputed with this infill point. The process is iteratively repeated until convergence, or until the maximum number of function evaluations allocated to this stage is reached.

\section{Trust Region Method}

In high dimensional, nonlinear functions (such as the case of those involving the presence of shock waves) the convergence of the previous stage is not always guaranteed. The trust region method is a local exploitation method used to find the local minimum in the region of influence of the current sampled minimum [33]. In order to converge towards a global minimum, a good balance between exploration and exploitation is required in the previous stage.

The optimum point in the surrogate is again found with differential evolution, but this time the search space is centered in the current real minimum, $y_{\text {min }}$, (sampled in the CFD) and bounded to a given trust region, $\delta$. The optimum location is validated in the Full Order Model, and the surrogate is updated. The next trust region $\delta_{m}$ is reduced if the surrogate is not accurate enough or increased otherwise. In case the new evaluated point is lower than the current sampled minimum, the center of the trust region is updated. Then, the process is repeated until convergence. The details of the implementation can be found in Forrester [22].

\section{B. Surrogate-Based Uncertainty Quantification}

The main problem in UQ is the propagation of the uncertainty through the numerical solver [13]. For the determination of both statistical moments and quantiles of the quantity of interest, QoI, the classical stochastic approach of Monte Carlo sampling is expensive. The solution to this problem is the use of an approximation of the QoI, which in this case is the drag coefficient. This approximation or surrogate is cheaper to evaluate through Monte Carlo [34] than the full order model. The surrogate-based uncertainty quantification (SBUQ) process follows the process represented in Fig. 6 This framework balances exploration (global representation of the QoI) with exploitation (focus on the value of the QoI closer to the quantile).

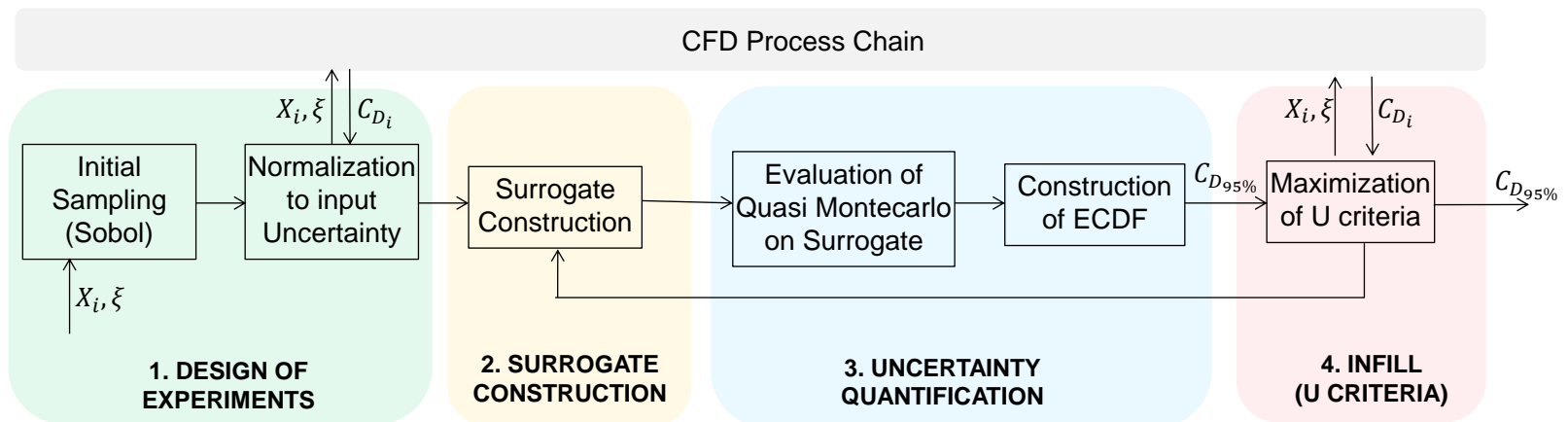

Fig. 6 Surrogate-based uncertainty quantification framework to efficiently characterize the quantile

\section{Design of Experiments}

Schillings [12] and Maruyama [15] have shown that for UQ, the accuracy of the surrogate can be improved if the initial sampling follows the distribution of the input uncertainties $\xi$. In the design of experiments, more samples should be placed along the mean, than along the tails of the input PDFs. Following this approach, when performing Monte Carlo on the surrogate with a large number of samples, the accuracy will be larger in the points that are evaluated more often, and lower in those regions that have less probability of being evaluated. As a result, for the problem at hand, an initial sampling based on Sobol sequences is scaled to the input distribution. The samples are evaluated in the non-intrusive CFD code in the framework introduced in Fig. 4

\section{Surrogate Construction}

Kriging models can not only be used in optimization, but also to approximate the stochastic landscape of the QoI at a given design $X_{i}$. This is due to their good adaptation to nonlinear functions and the availability of the error estimator [35]. Based on the initial sampling, the Kriging model is constructed following the same steps as in subsection III.A.2 


\section{Evaluation of statistics}

The evaluation of the QoI in the surrogate is done through the direct integration of 1,000,000 Quasi Monte-Carlo samples. Although the surrogate provides a fast evaluation of the response (drag coefficient), the convergence rate of Quasi Monte Carlo is faster than that of classical Monte Carlo. As a result, the sampling is predefined (derandomized algorithm) through Sobol Sequences [26] and the stochastic space is evaluated more efficiently.

The QoI is the given $\tau$ quantile. It is the value below which the drag (a random variable) is obtained with a probability of $\tau \%$. With a high quantile $(\tau=95 \%)$, this approach is preferred to classical min-max problems, as these are often too conservative [36].

To obtain the quantile, the Empirical Cumulative Distribution Function (ECDF) [37] is computed from the Quasi Monte Carlo sampling on the Kriging meta-model. This approach has been used in the past to evaluate the quantile of the drag in optimization under uncertainty problems involving airfoil design [36].

$$
\mathrm{ECDF}_{n}(x)=\frac{\text { number of elements in sampling } \leq \mathrm{x}}{n}=\frac{1}{n} \sum_{i=1}^{n} I\left(x_{i} \leq x\right)
$$

where $\mathrm{n}$ is the number of samples evaluated through Quasi Monte Carlo, and $I$ is the indicator function with two possible values: 1 if the event inside the brackets occurs and 0 if not:

$$
I\left(x_{i} \leq x\right)=\left\{\begin{array}{lll}
1, & \text { if } & x_{i} \leq x \\
0, & \text { if } & x_{i}>x
\end{array}\right.
$$

By using the surrogate to evaluate the statistics, the number of samples is large enough and the ECDF converges to the Cumulative Distribution Function, CDF, according to the Glivenko-Cantelli theorem [38]. By numerically inverting the $\mathrm{CDF}$ it is possible to obtain any given $\tau$ quantile.

\section{Active Infill Criterion}

The active infill criterion follows the U-function [39] and equivalent probability of misclassification $P_{M}$ [40]. At any given point, $\xi$, where the Kriging mean value is lower than the current quantile using the surrogate, $\hat{y}(\xi)<\hat{q}_{\tau}$, it is possible to obtain the probability of exceeding this quantile value, $P_{M}=P\left(\hat{y}(\xi)>\hat{q}_{\tau}\right)$. The opposite, the probability of overestimating the quantile given that the Kriging mean value is higher than the quantile, can also be obtained. Then, the probability of misclassification is defined as:

$$
P_{M}=\min \left\{\Phi\left(\frac{\hat{y}(\xi)-\hat{q}_{\tau}}{\hat{\sigma}(\xi)}\right), \Phi\left(\frac{\hat{q}_{\tau}-\hat{y}(\xi)}{\hat{\sigma}(\xi)}\right)\right\}=\Phi\left(\frac{\left|\hat{q}_{\tau}-\hat{y}(\xi)\right|}{\hat{\sigma}(\xi)}\right)
$$

Locations where the probability is maximum will have a strong influence on the predicted quantile by the surrogate, and new samples should be added at this location [41]. The probability of misclassification is maximum when the fraction $\frac{\left|\hat{q}_{\tau}-\hat{y}(\xi)\right|}{\hat{\sigma}(\xi)}$ is minimum. As a result, it is equivalent to minimizing the so called $\mathrm{U}$ function:

$$
U(\xi)=\frac{\left|\hat{q}_{\tau}-\hat{y}(\xi)\right|}{\hat{\sigma}(\xi)}
$$

Then, the location of the new sample point is found by:

$$
\xi^{*}=\operatorname{argmax} P_{M}(\xi)=\operatorname{argmin} U(\xi)
$$

A new surrogate model is constructed with this additional sample and a new location is iteratively found following equation 12 until the required level of accuracy is achieved.

The infill criterion balances exploitation with exploration by sampling points closer to the calculated quantile or with a high error. The quantile value is calculated with high accuracy at a relatively low cost. This approach is especially useful when dealing with high quantiles, which would otherwise require a large number of function evaluations to achieve the required accuracy. 


\section{Bi-Level Surrogate-Based for Optimization under with Uncertainty}

The bi-level surrogate model is developed by combining the previously introduced surrogate-based optimization and surrogate-based uncertainty quantification processes as shown in Fig. 7 The outer level takes into account the optimization which is dependent only on the design variables $X$ and has as output the statistic of the QoI. The inner level is responsible for the uncertainty quantification process of the QoI at a given design point $X_{i}$ with uncertainty $\xi$. In the proposed application, the quantity of interest is the drag coefficient, and its statistic is the $\tau$ quantile of the drag.

This approach focuses on the refinement of the region where the robust solution is located. As a result, the efficiency over other optimization algorithms is improved, as will be shown in Section IV.A.

Another advantage is the parallelization of the DoE stage of the UQ level. As the initial samples are defined beforehand, these can all be computed at the same time. In addition, as the CFD solver allows for domain decomposition, an additional level of parallelization is also possible.

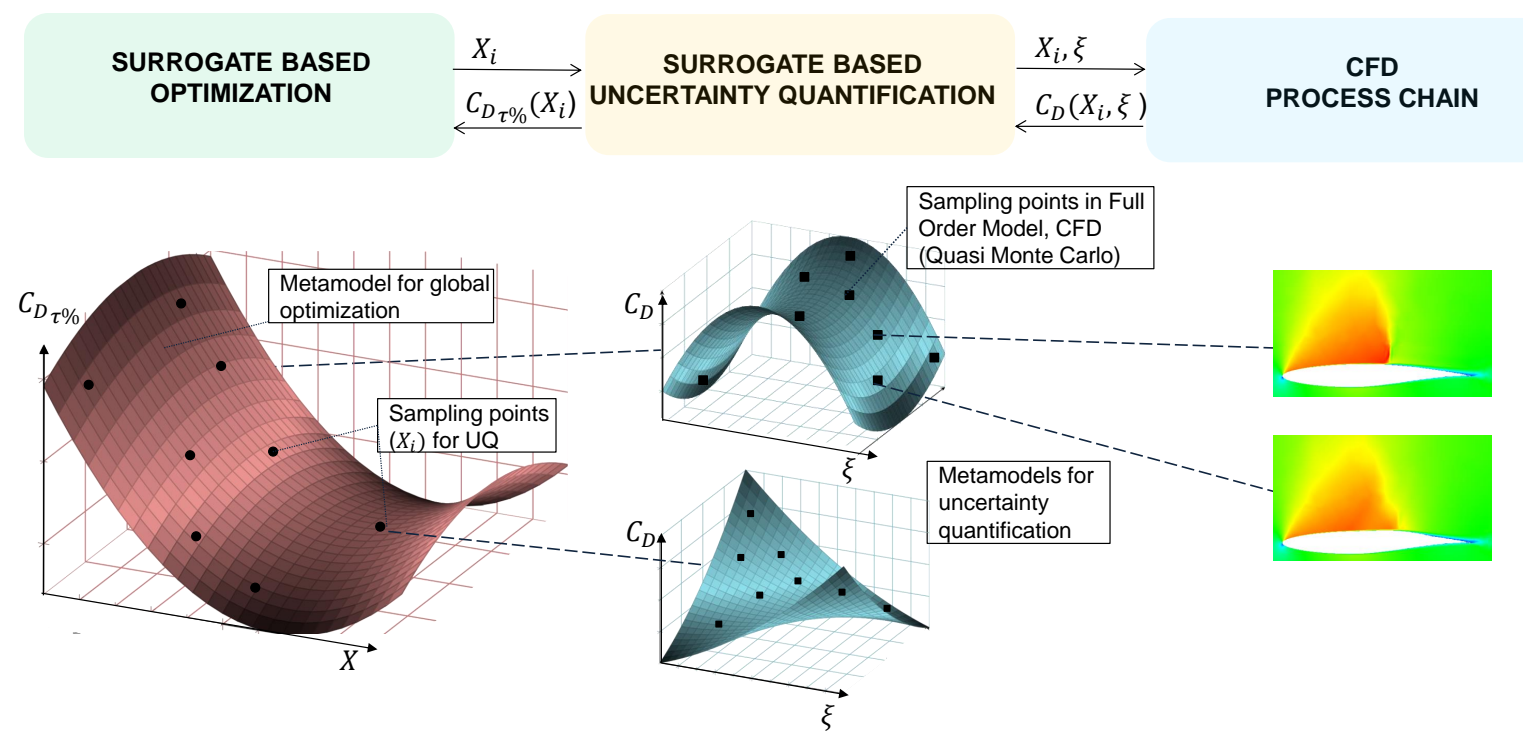

Fig. 7 Bi-level approach for uncertainty quantification; Surrogate of statistics (left); Surrogate of random variable (middle); Full order model evaluation (right)

\section{Results}

This section presents the results for the deterministic and the robust optimizations of the SCB retrofitted to the RAE2822 using the framework previously described. It also provides the validation of the statistics of the quantity of interest by comparing the surrogate-based UQ with direct integration.

\section{A. Deterministic Optimization}

The deterministic optimization problem of equation 4 has been computed following three optimizers, with the objective of assessing the global accuracy and the time savings of the surrogate-based optimization approach:

1) A Differential evolution algorithm [32] is used as a reference with an initial population size of 30 individuals (6 times the number of design parameters). It is the same algorithm as the one used for obtaining the minimum value on the surrogate in the SBO approach, but this time the full order model is being evaluated.

2) Subplex, a gradient-free method of Rowan [42], effectively decomposes the design space into low dimensional subspaces and searches for the convex hull. It is more efficient than the Simplex method by linearly scaling with the dimensionality and can be applied to noisy functions. Two convergence criteria based on the difference in the objective function and on the difference in the design vector between two consecutive iterations is defined.

3) The SBO method presented in section III.A

Figure 8 shows the optimization history of the normalized drag coefficient for the three approaches against the optimization iteration number. It is assumed that the optimum found through differential evolution, after 1140 iterations, is the global one. Then, the SBO approach is able to find an optimum solution very close to this global optimum at a 
tenth of a cost of iterations (or function evaluations). Also, SBO finds a better optimum than the Subplex method with half the iterations. Requiring much less function evaluations, SBO explores the design landscape very efficiently.

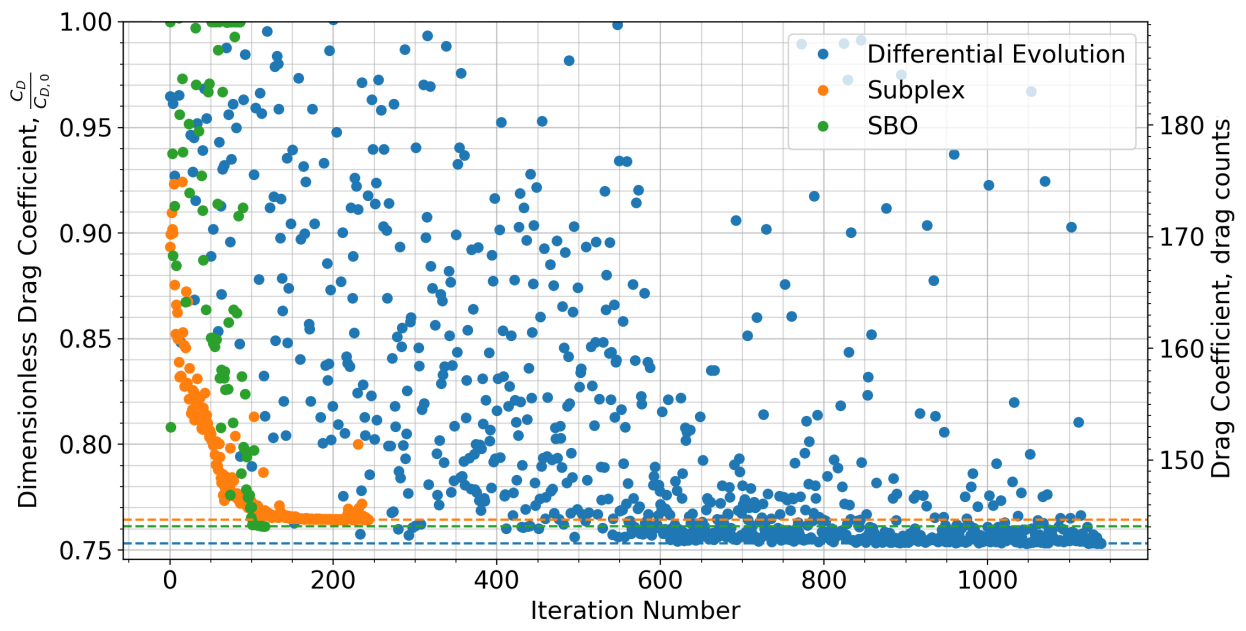

Fig. 8 Convergence history of the drag coefficient for deterministic optimization

The results of the three different optimization runs are summarized in Table 3 in terms of optimum geometry, drag reduction and required number of iterations. In the three cases, the optimum bump extends all the way to about $80 \%$ of the chord of the airfoil, which is close to the hinge point of the hypothetical flap defined in the constraint.

Table 3 Deterministic optimization results

\begin{tabular}{c|c|c|c|c|c|c|c|c}
\multirow{2}{*}{ Optimizer } & \multicolumn{5}{|c|}{ Optimum Geometry } & \multicolumn{2}{c|}{ Drag Reduction } & Function \\
\cline { 2 - 8 } & $h_{\text {bump }}$ & $x_{h_{\text {bump }}}$ & $l_{\text {bump }}$ & $x_{\text {start }}$ & $t_{\text {bump }}$ & Percentage & Drag counts & Evaluations \\
\hline Diff. Evolution & 0.009 & 0.644 & 0.45 & 0.35 & 1.282 & $24.694 \%$ & 46.74 & 1140 \\
Subplex & 0.008 & 0.555 & 0.374 & 0.426 & 1.237 & $23.56 \%$ & 44.59 & 244 \\
SBO & 0.0077 & 0.598 & 0.402 & 0.398 & 1.111 & $23.87 \%$ & 45.18 & 116
\end{tabular}

Physically, the bump reduces the wave drag by replacing the near-normal shock wave with an isentropic compression wave, as shown in Fig 9 for the optimum obtained with SBO. The total drag reduction is almost $25 \%$, as the shock control bump is efficient in smearing the strong initial shock wave.
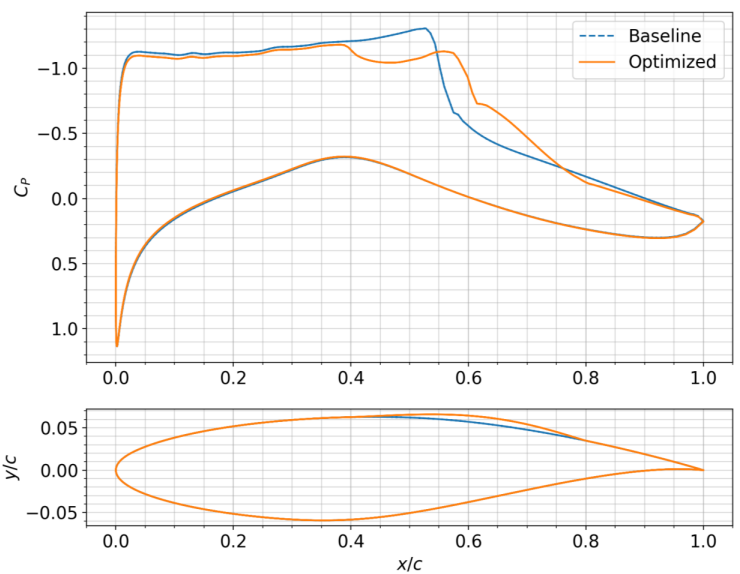

a)

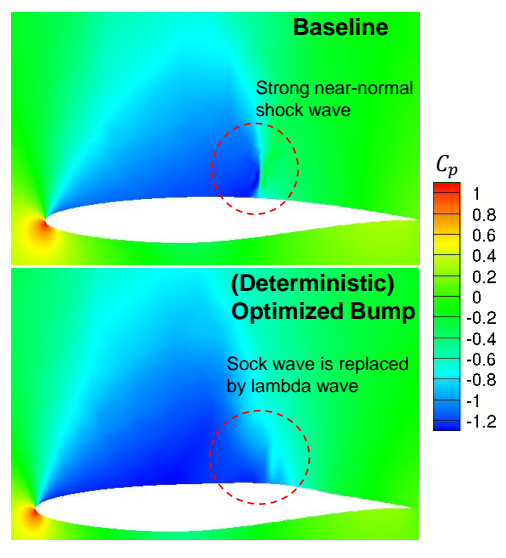

b)

Fig. 9 Baseline and deterministic configurations; a) Surface pressure coefficient; b) Field pressure coefficient 


\section{B. Validation of Uncertainty Quantification (I)}

Following Figure 6 , for an accurate estimation of a high quantile using the surrogate, it is necessary to determine the proper number of samples for the DoE and infill stages. The uncertainty quantification framework is validated on the deterministic optimum shape obtained with SBO under operational and geometrical uncertainties. The reference $95 \%$ quantile of the drag, $C_{D_{95 F_{\circ}}}$, is obtained with 10,000 Quasi Monte Carlo realizations of the full order model (CFD).

Figure 10 shows the convergence history of the surrogate based uncertainty quantification using different sampling strategies. These consist in different proportions between the DoE (exploration) and infill (exploitation). The required accuracy is set to 0.3 drag counts. This is in line with the minimum improvement in the performance required for a designer in order to consider one configuration over the other.

As expected, the error in the $95 \%$ quantile is reduced as the number of samples used in the construction of the surrogate increases. However, the use of training samples following only a global DoE approach (pink line) is not enough to reduce the quantile error to the required level of accuracy. The active infill criterion is effective in further reducing the error. A clear trade-off is present. Without an accurate global representation of the stochastic space provided by the DoE, the initial location of the quantile can not be well estimated.

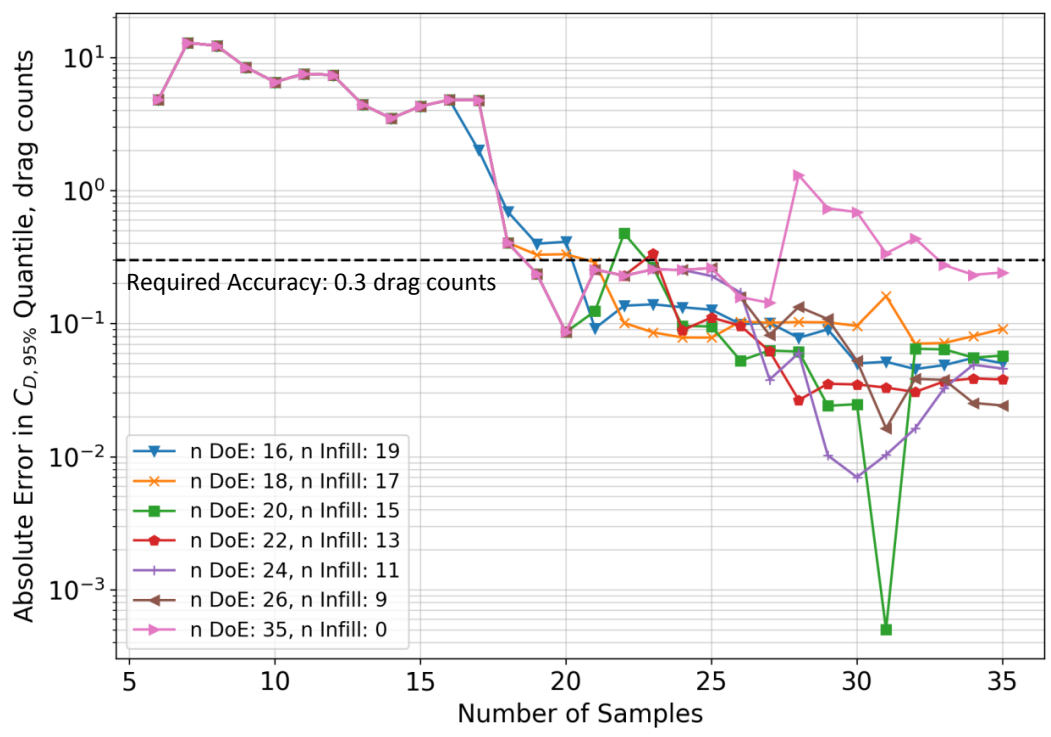

Fig. 10 Convergence error of drag quantile, $C_{D_{95 \%}}$, for deterministic optimum configuration under different sampling strategies. Reference solution obtained with 10,000 Quasi Monte Carlo realizations

Based on this study, at each iteration of the optimization in the outer level, a surrogate is constructed in the stochastic space with $24 \mathrm{DoE}$ samples and 5 additional infill samples following the $\mathrm{U}$ criterion. This guarantees a high accuracy in the estimation of the statistic, which is critical in the case of high quantiles.

The resulting uncertainty space with this sampling strategy is shown in Figure11. The drag coefficient is represented as a function of the uncertain lift coefficient and Mach number, for the nominal bump height. After drawing Monte Carlo samples based on the surrogate (small black dots), the $95 \%$ quantile of the drag is obtained (black line). The quantile divides the $5 \%$ of the realizations with highest drag, while keeping below the $95 \%$ remaining with lower drag. Note how the infill samples are distributed along this quantile, while the DoE samples are focused on the locations where the realizations are most common.

\section{Robust Solutions}

Once the capabilities of the framework have been demonstrated in terms of the deterministic optimization and the uncertainty quantification, the optimization under uncertainty of the shock control bump is performed through the bi-level surrogate approach. Two different optimum configurations, one in terms of the $50 \%$ quantile of the drag coefficient and other in terms of the $95 \%$ quantile, are respectively found.

Figure 12 shows the convergence history of the robust optimization. The objective function, the $95 \%$ quantile of the drag coefficient, is represented in black squares, while the other colors represent the five normalized design variables at 


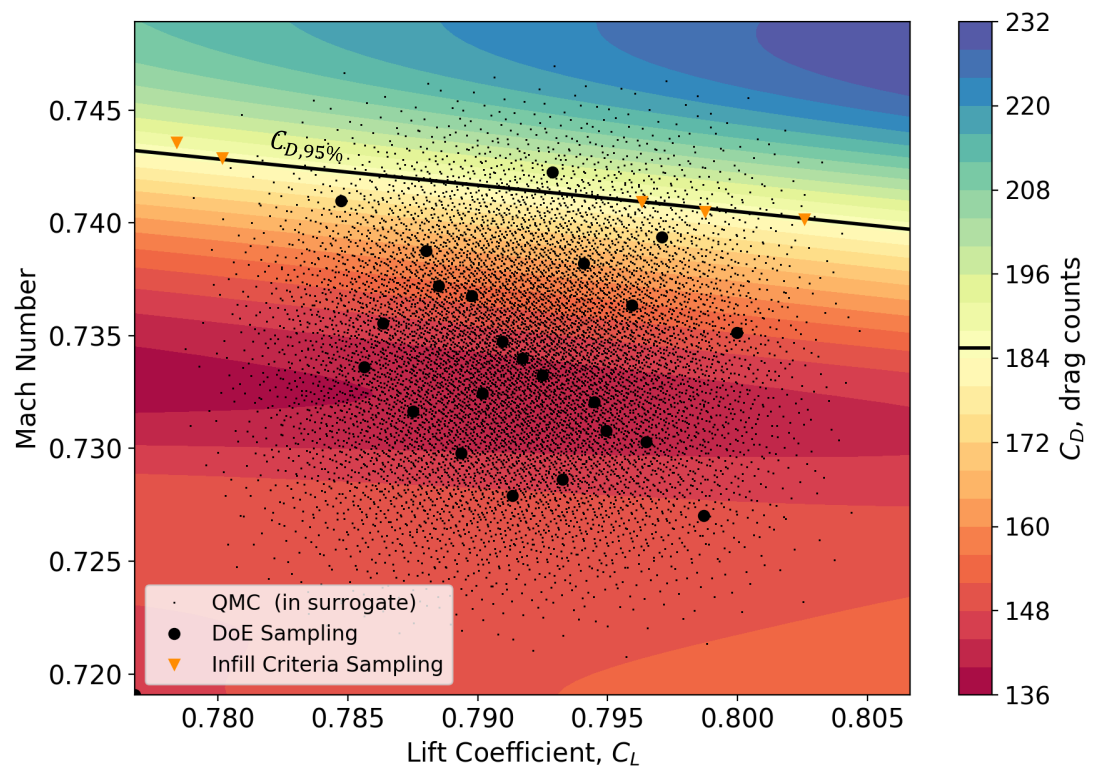

Fig. 11 Drag field as a function of stochastic operating conditions and zero uncertainty in bump height. Results for deterministic optimum configuration

each iteration. These range from one, the upper limit in table 1 , to zero, the lower limit. Highlighted markers represent the current optimum configuration until a better one is found.

After the initial DoE and EI phases, the optimum solution stabilizes, and is further refined through the trust region approach. The total number of CFD evaluations is 3219 (the stochastic space is analyzed 111 times with 29 CFD evaluations in each iteration for the surrogate-based uncertainty quantification). The parallelization strategy considerably reduces the computational time.

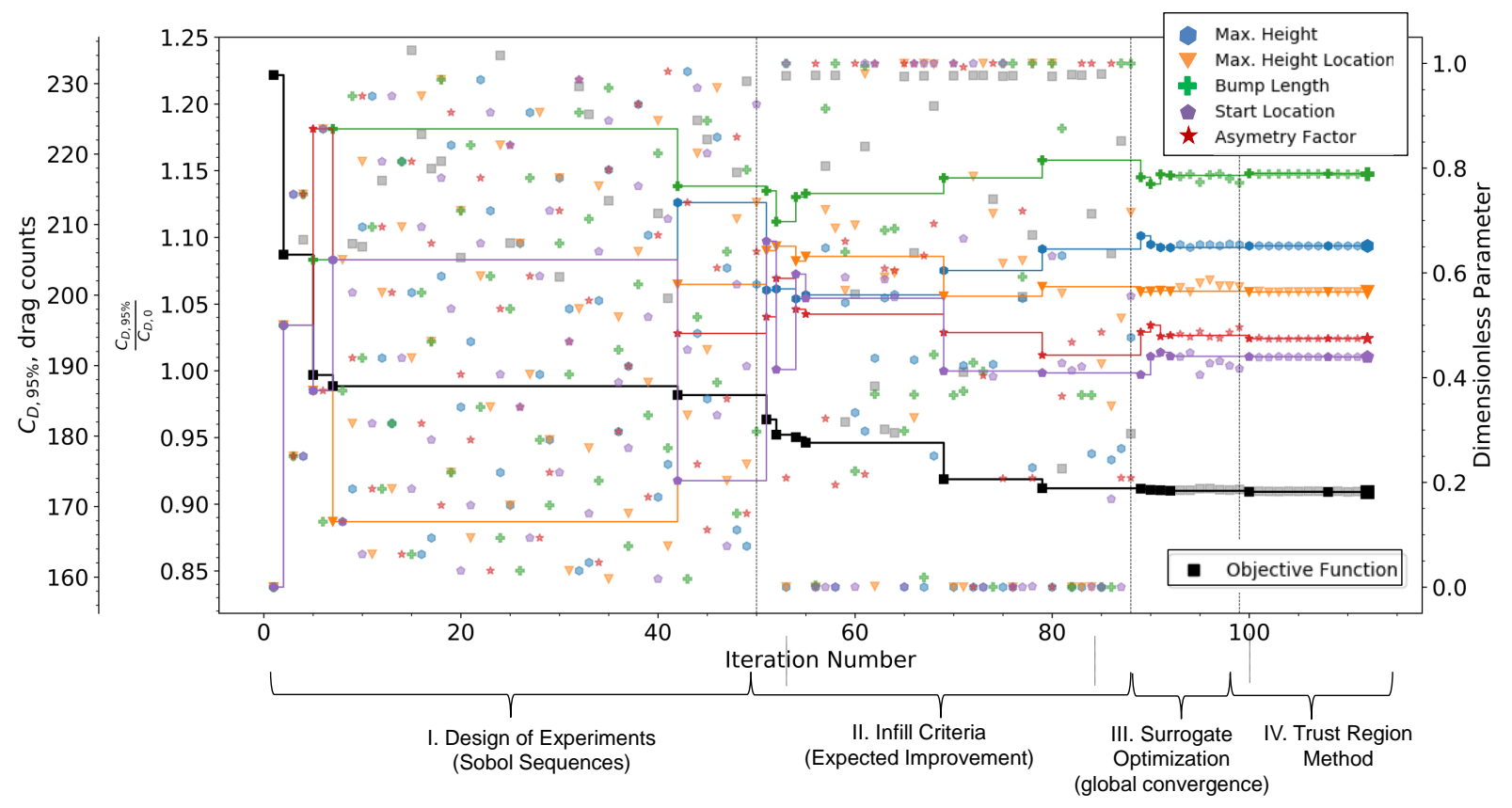

Fig. 12 Convergence history of robust optimization. $C_{D_{95 \%}}$ and design parameters vs iteration number 
When uncertainty is introduced, the drag coefficient becomes a random variable. Figure 13 shows the histograms of the two different robust optimum, of the deterministic optimum and of baseline airfoil without bump. The 50\% and 95\% quantiles are also shown for each geometry.

The optimum robust bump geometry with focus on the $95 \%$ quantile (blue histogram) provides a decrease in the 95\% quantile of the drag by $25.5 \%$ (58.9 drag counts) with respect to the baseline RAE2822 airfoil where the bump is not present (red histogram). This configuration is able to deal better with the uncertainties under off-design conditions making it also more robust than the bump shape obtained in the deterministic optimization (orange histogram). In particular, it reduces the $95 \%$ quantile by $6.7 \%$ (12.4 drag counts) compared to the deterministic counterpart. This is due to the increase in bump height. By increasing the bump height, the SCB is more effective alleviating strong shock waves at high velocities and lift coefficients. As a result, its histogram has the shortest tail.

The main drawback of the robust shock control bump with focus in the $95 \%$ quantile of drag is its higher mean drag compared to deterministic optimum configuration. This high bump is not effective when alleviating the drag at nominal operating conditions.

To deal with this problem, the configuration with the optimum 50\% quantile displaces most of the weight of its PDF to the left. As expected, it is the configuration with the lowest median ( 2.5 drag counts lower than the deterministic configuration) and mean. This comes at the expense of a longer tail, and worst off-design conditions. This configuration has the worst $95 \%$ quantile (excluding the baseline configuration). However, it is an appealing design when the dealing with day to day events operational, and when off design conditions are not critical for the performance.

The deterministic solution presents (as with the optimum 50\% quantile configuration), a strong positive skewness. The deterministic shock control bump is very effective around the deterministic design point (Mach number, lift coefficient and shape constant). When geometrical and operational uncertainties are present, the performance is strongly deteriorated from these nominal conditions even at lower lift coefficients or freestream Mach numbers, where the drag is usually reduced.

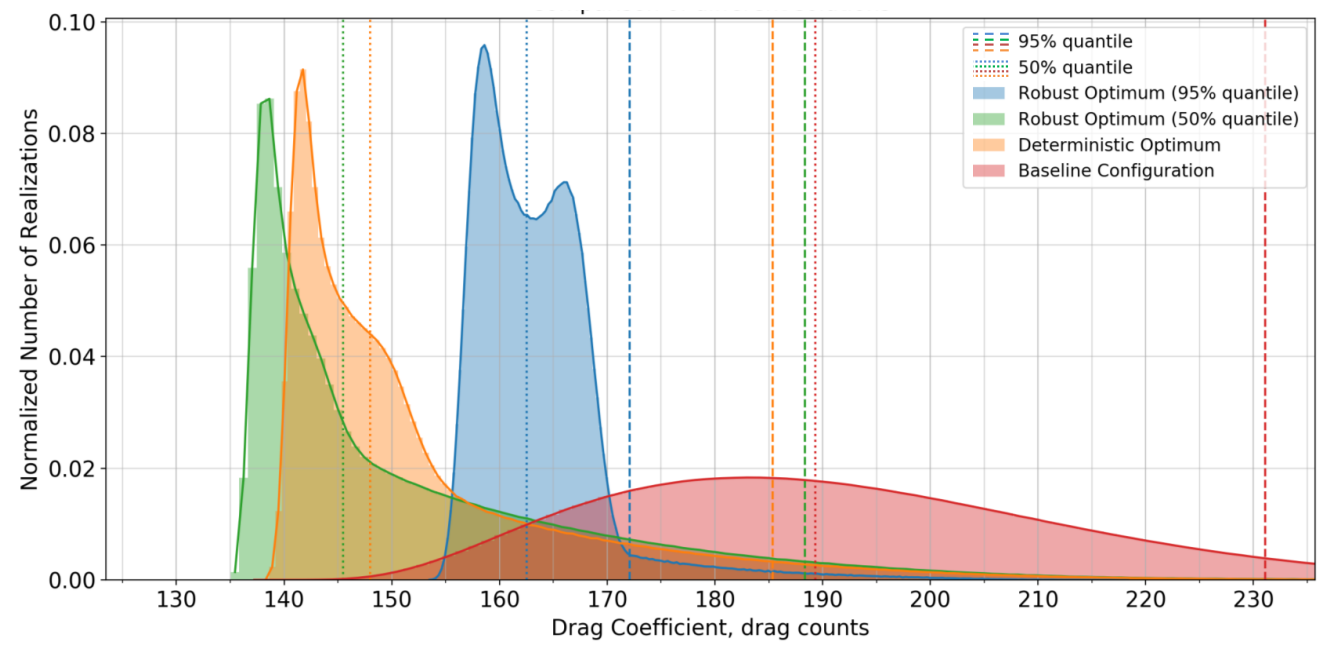

Fig. 13 Histograms of the uncertain drag coefficient for configurations at hand: $95 \%$ robust optimum, 50\% robust optimum, deterministic optimum and baseline configurations

A complete overview of the two different robust configurations is shown in Table 4 in terms of the design vector and quantiles. For comparison, the characteristics of the baseline and deterministic optimum configurations are also shown. In Figure 14 the two optimum bump geometries are also represented. The optimum bump with focus on the 95\% quantile is the highest and is located the furthest downstream.

To physically understand the results of Fig $13,10,000$ stochastic realizations for each of the optimum configurations have been obtained in the full order model following Quasi Monte Carlo. The pressure coefficient of 100 of these realizations along the airfoil surface is shown in Fig 15 , represented by grey lines. This is done for the robust optimum under the 50\% quantile (left) and the robust optimum for the $95 \%$ quantile (right). Locations with the largest concentration of grey lines represent the most common scenarios. The thick lines represent those realizations (from the 10,000 initial samples) equivalent to the $5 \%, 50 \%$ and $95 \%$ quantile. 
Table 4 Robust Optimization Results

\begin{tabular}{l|c|c|c|c|c|c|c}
\multirow{2}{*}{ Configuration } & \multicolumn{5}{|c|}{ Optimum Geometry } & \multicolumn{2}{c}{$\begin{array}{c}\text { Quantile } \\
\text { (drag counts) }\end{array}$} \\
\cline { 2 - 8 } & $h_{\text {bump }}$ & $x_{h_{\text {bump }}}$ & $l_{\text {bump }}$ & $x_{\text {start }}$ & $t_{\text {bump }}$ & $C_{D_{95 \%}}$ & $C_{D_{50 \%}}$ \\
\hline Baseline & 0 & 0 & 0 & 0 & 0 & 231.1 & 189.3 \\
Deterministic Optimum & 0.00772 & 0.598 & 0.402 & 0.398 & 1.11 & 185.4 & 148 \\
$95 \%$ Optimum & 0.00977 & 0.654 & 0.386 & 0.414 & 0.991 & 172.1 & 162.5 \\
$50 \%$ Optimum & 0.00726 & 0.619 & 0.432 & 0.369 & 1.129 & 188.5 & 145.5
\end{tabular}

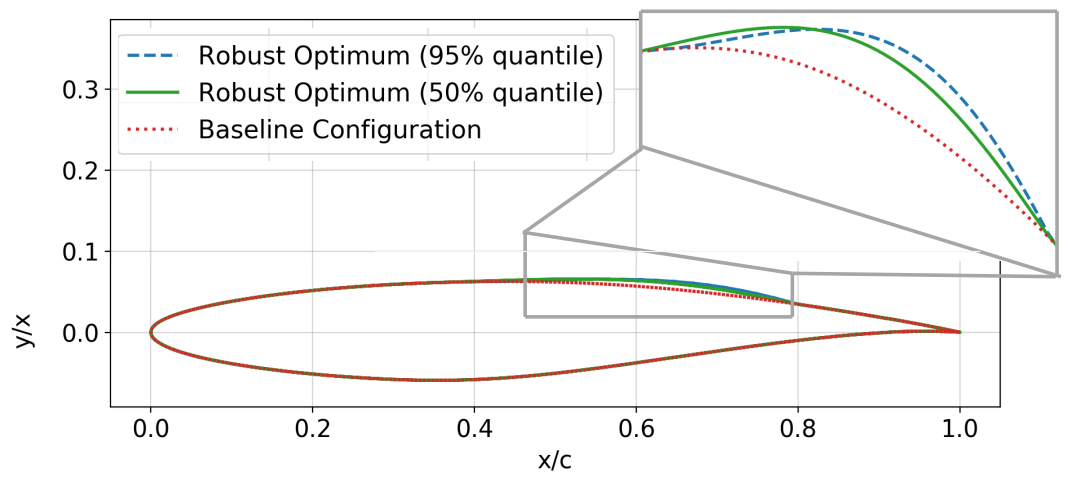

Fig. 14 Geometries of baseline and robust optimum configurations

It can be deduced from the pressure field that the robust configuration aiming at the $50 \%$ quantile has the shock wave in the ideal location during most of the time. This is in line with the positive skewness of its histogram and its low mean drag. However, at off-design conditions, the performance rapidly deteriorates. In its $95 \%$ realization a strong normal shock wave is present (black line). As expected, this shock wave is stronger than the one from the robust optimum focused on off-design conditions. This explains its large 95\% quantile value and longer tail of the histogram.

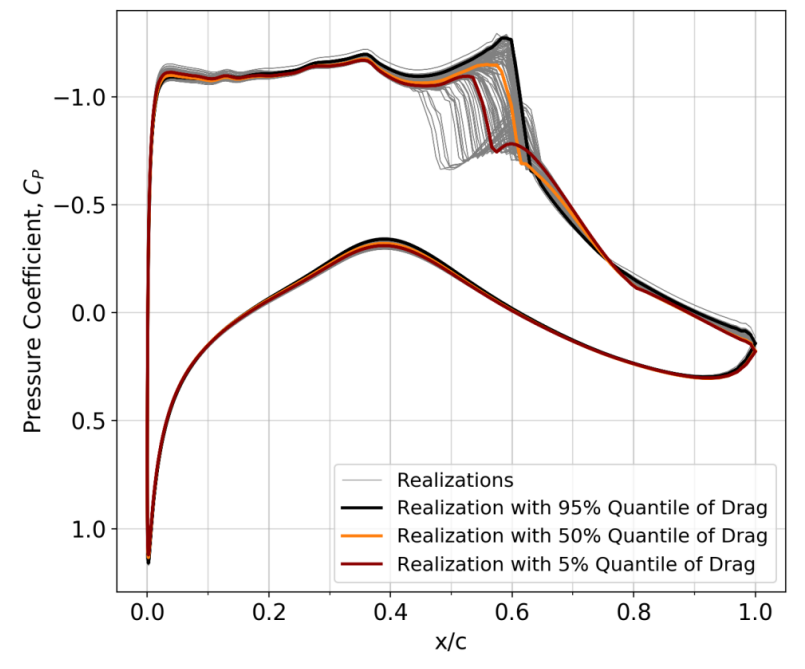

a)

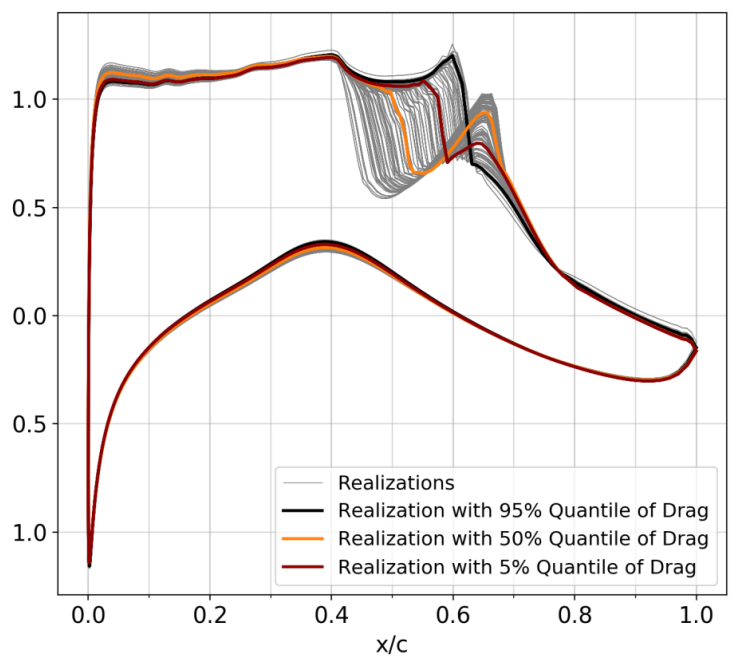

b)

Fig. 15 Realizations of the pressure coefficient due to uncertainty. Highlighted the realizations corresponding to $5 \%, 50 \%$ and $95 \%$ quantile of drag. a) Optimum Shape in $C_{D, 50 \%}$; b) Optimum robust shape in $C_{D_{95 \%}}$ 
On the other hand, the robust bump with focus on the $95 \%$ quantile presents a weaker normal shock wave at off-design conditions motivated by the increase in bump height. In addition, the bump is located further downstream and is shorter, increasing its effectivity at higher Mach numbers. However, as previously introduced, this increase in height deteriorates the behaviour around the 50\% quantile. In this case, at medium to low Mach numbers and lift coefficients, an initial normal shockwave is followed by an expansion wave as the flow is further accelerated along the bump. This leads to a secondary normal shockwave. As a result, the shock wave location is longitudinally more uniformly distributed with respect to the first configuration. With the increase in Mach number and lift coefficient, the initial shock wave moves forward and the expansion and secondary shock wave decrease until disappearing at around the $95 \%$ quantile. This configuration presents a good trade-off between nominal and off design conditions.

The pressure coefficient field for the 50\% and $95 \%$ realizations, as well as the mean and standard deviation are shown in Figure 16 for the baseline airfoil and two robust optimum configurations. As expected and previously explained, the robust configuration with focus in the $95 \%$ quantile is most effective in smearing the shock wave in the $95 \%$ realization.

The mean pressure field has no physical meaning, but helps in understanding the average behaviour under uncertainties. For example, the optimum configuration with focus in the $50 \%$ quantile has during most of the realizations a smeared shockwave (softest transition from negative to positive pressure coefficients). In opposition, the robust configuration with focus in the $95 \%$ quantile shows a secondary shock wave in order to better withstand off design conditions. Finally, the baseline configuration always presents a strong shock wave.

From the standard deviation pressure field, it is shown that most of the uncertainty occurs around the foot of the $\mathrm{SCB}$, at the location of the main shock wave. The longitudinal displacement of the shock wave due to the uncertainties is largest in the Optimum configuration with focus in the $95 \%$ quantile. However, this longitudinal displacement makes the quantile more robust w.r.t. off design conditions, specially compared to the baseline configuration. In this latest case, the longitudinal displacement of the shock wave is the shortest, at an expense of a stronger shock wave.

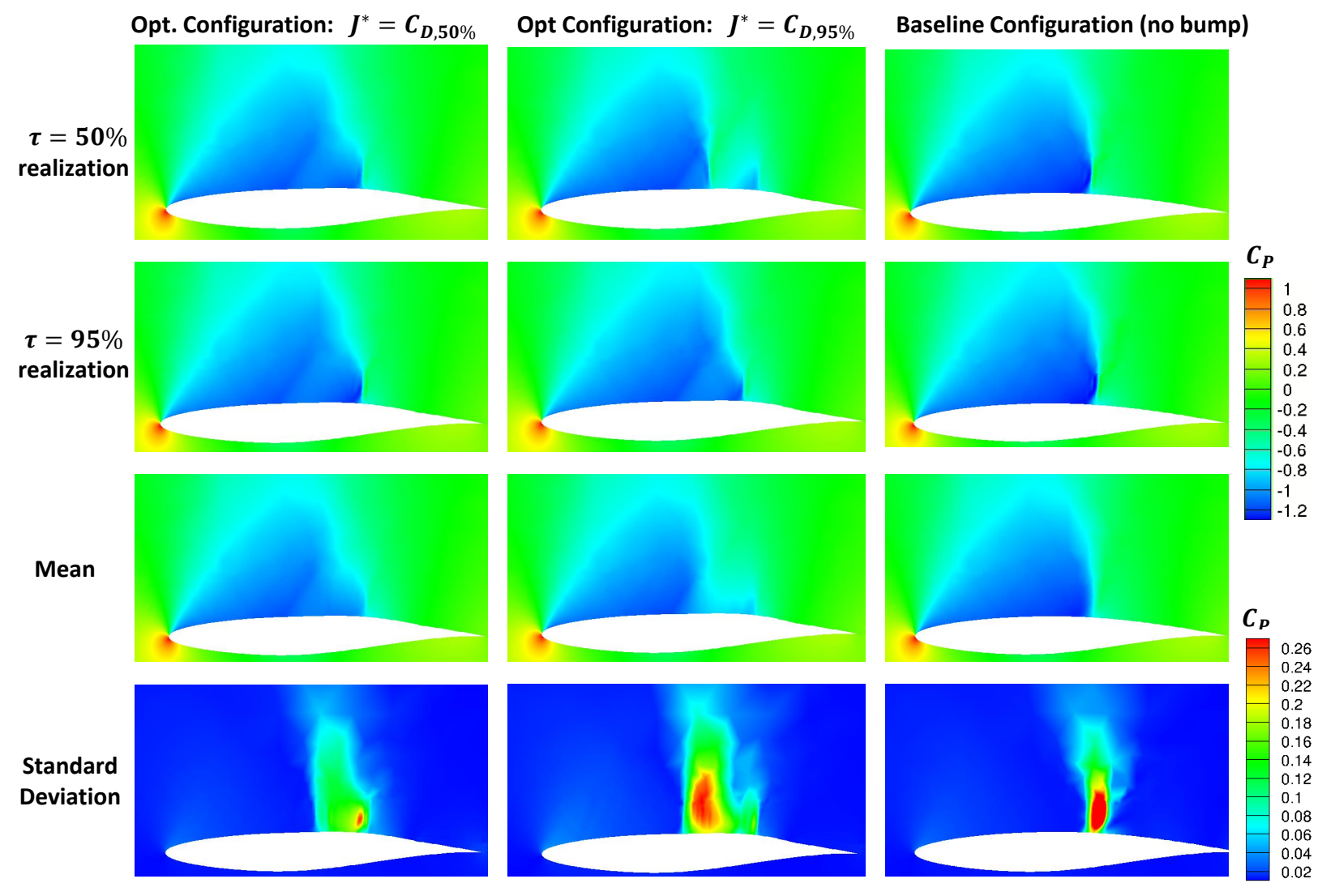

Fig. 16 Pressure coefficient field for robust and baseline configurations. 50\% and $95 \%$ realizations, mean value and standard deviation value 


\section{Validation of the Uncertainty Quantification (II)}

The optimum results were obtained by the surrogate-based uncertainty quantification framework where only 29 CFD samples were used to estimate the quantile in each iteration. Then, the given quantile of each optimum configuration have also been validated by computing 10,000 realizations using the full order model following Quasi Monte Carlo Sampling. Table 5 shows the absolute and relative error of the quantile of the QoI for the optimum configurations. Overall, it has been found that using the proposed approach, the error in the quantile obtained with the surrogate is smaller than the initial requirement of 0.3 drag counts.

As shown in Fig. 17, the reference Empirical Cumulative Distribution Function, obtained with 10,000 samples, is equivalent to the one obtained with the surrogate built with 29 training samples. When no surrogate is present, the ECDF can be obtained from direct integration with equivalent 29 samples. In this case, a large error in the quantiles is present. As a result, the surrogate enhances the accuracy of the uncertainty quantification making possible a good estimation of the quantile.

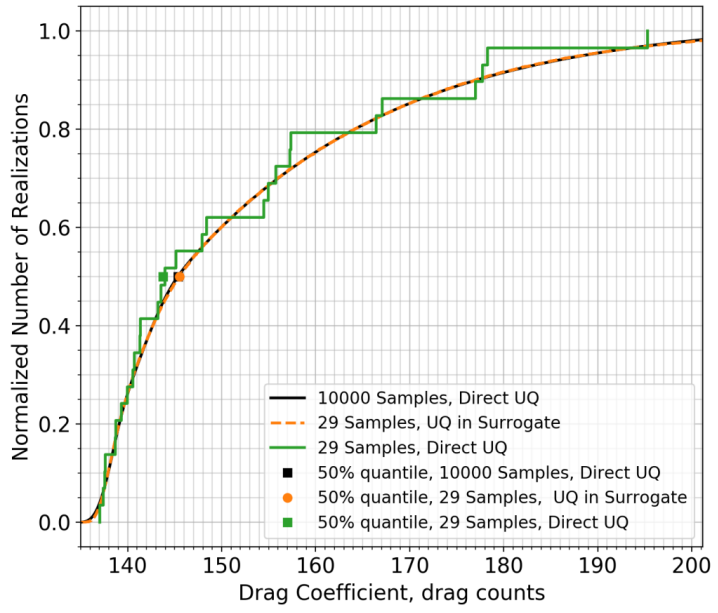

a)

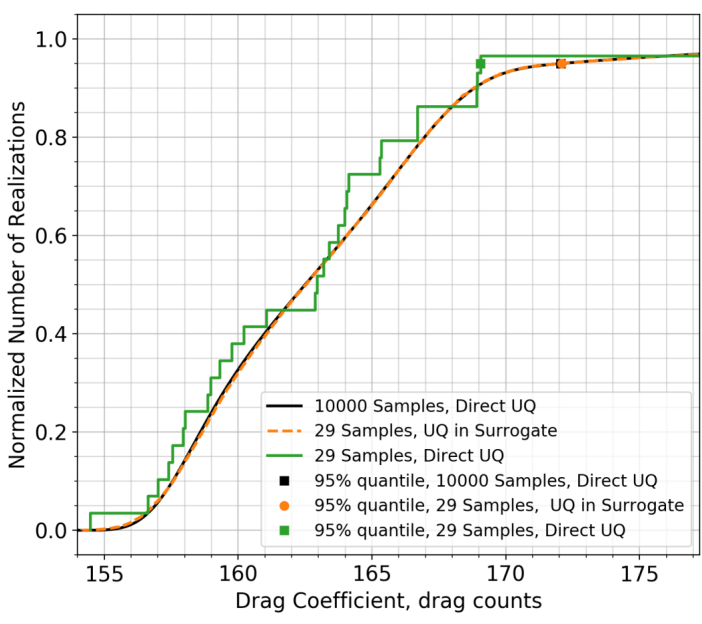

b)

Fig. 17 Comparison of Empirical Cumulative Distribution Functions for direct integration with 29 samples (with and without surrogate) and 10,000 samples: a) Robust optimum on $C_{D_{50 \%}}$; b) Robust optimum on $C_{D_{95 \%}}$

Table 5 Error in surrogate-based uncertainty quantification

\begin{tabular}{c|c|c|c|c}
\multirow{2}{*}{ Configuration } & \multicolumn{2}{|c|}{ Absolute Error (drag counts) } & \multicolumn{2}{c}{ Relative Error } \\
\cline { 2 - 5 } & $50 \%$ Quantile & $95 \%$ Quantile & $50 \%$ Quantile & $95 \%$ Quantile \\
\hline $50 \%$ Optimum & 0.18 & 0.097 & $0.125 \%$ & $0.05 \%$ \\
$95 \%$ Optimum & 0.04 & 0.023 & $0.024 \%$ & $0.013 \%$
\end{tabular}

\section{Conclusions}

A framework for the efficient aerodynamic shape optimization under uncertainty has been presented, validated and applied to the robust design of shock control bumps. The bi-level surrogate approach combining a surrogate-based optimization with surrogate-based uncertainty quantification is able to effectively explore both design and stochastic landscapes.

The surrogate-based optimization combines an initial sampling scheme based on Sobol sequences with a Kriging surrogate model, an adaptive sample refinement strategy following the maximum expected improvement criterion, an iterative evaluation of the global minimum of the surrogate with a differential evolution algorithm, and a trust region method. Due to the good balance between exploration and exploitation, the surrogate based optimizer efficiently finds the global optimum, while requiring an order of magnitude less function evaluations than traditional global optimization approaches. 
The uncertainty quantification, performed at every iteration of the surrogate-based optimization, is based on a Kriging meta-model and an active infill criterion. It is able to reproduce the reference cumulative distribution function and the quantile of interest of the optimum configurations with high accuracy. In this case, a trade-off is present between the required global refinement of the stochastic space and the local refinement in areas close to the quantile. Following this approach, the determination of any given $\tau$ quantile of the quantity of interest can be computed with considerably less function evaluations than with direct integration through Quasi Monte Carlo.

When accounting for operating and geometrical uncertainties, the use of a robust optimization framework with focus on the quantile of the airfoil drag coefficient is useful in the determination of a robust shock control bump. If the interest is the minimization of extreme events, the optimization of the $95 \%$ quantile of drag is considered. The resulting configuration has the highest bump height that smears the normal shock wave at high values of lift and Mach, around the $95 \%$ quantile. This robust configuration is able to better withstand operating and geometrical uncertainties than the configuration obtained with deterministic optimization. It distributes the longitudinal location of the shock wave more uniformly at an expense of a secondary shock wave at the end of the bump at nominal conditions.

The secondary shock wave can be removed if optimizing for the median or 50\% quantile. In this case, most common events correspond to a lower value of drag due to the weaker normal shock wave over the bump. However, this configuration is not as effective as the previous one in reducing the drag at off-design conditions.

By reducing both the number of iterations in the optimization stage and the number of CFD evaluations in the uncertainty quantification, it is possible to come up with an efficient process chain within a reduced CPU time. In the future, the framework will be applied to the robust design of high dimensional aerodynamic shape optimization

problems involving natural laminar flow wings and three dimensional shock control bumps. Finally, an extension of the framework to a global surrogate accounting for both uncertainties and design parameters in a single model will be explored.

\section{Acknowledgments}

This work is funded by the European Commission's H2020 programme, through the UTOPIAE Marie Curie Innovative Training Network, H2020-MSCA-ITN-2016, Grant Agreement number 722734.

\section{References}

[1] Stanewsky, E., Délery, J., Fulker, J., and de Matteis, P., "Synopsis of the Project EUROSHOCK II," Drag Reduction by Shock and Boundary Layer Control, edited by E. Stanewsky, J. Délery, J. Fulker, and P. de Matteis, Springer Berlin Heidelberg, Berlin, Heidelberg, 2002, pp. 1-124.

[2] Ogawa, H., Babinsky, H., Pätzold, M., and Lutz, T., "Shock-Wave/Boundary-Layer Interaction Control Using Three-Dimensional Bumps for Transonic Wings,” AIAA Journal, Vol. 46, No. 6, 2008, pp. 1442-1452. doi:10.2514/1.32049.

[3] Ashill, F. J., P.R., and Shires, J., "A Novel Technique for Controlling Shock Strength of Laminar-Flow Airfoil Sections," Proceedings of the 1st European Forum on Laminar Flow Technology, Hamburg, 1992, pp. 175-183.

[4] McGowan, A., "AVST morphing project research summaries in fiscal year 2001," Tech. Rep. nasa tm-2002-2 11769, NASA, Aug. 2002.

[5] Bruce, P. J. K., and Babinsky, H., "Experimental Study into the Flow Physics of Three-Dimensional Shock Control Bumps," Journal of Aircraft, Vol. 49, No. 5, 2012, pp. 1222-1233. doi:10.2514/1.c031341.

[6] Lee, D. S., Periaux, J., Onate, E., Gonzalez, L. F., and Qin, N., "Active Transonic Aerofoil Design Optimization Using Robust Multiobjective Evolutionary Algorithms," Journal of Aircraft, Vol. 48, No. 3, 2011, pp. 1084-1094. doi:10.2514/1.c031237.

[7] Paetzold, M., Lutz, T., Kramer, E., and Wagner, S., "Numerical Optimization of Finite Shock Control Bumps," 44th AIAA Aerospace Sciences Meeting and Exhibit, American Institute of Aeronautics and Astronautics, 2006. doi:10.2514/6.2006-1054.

[8] Jinks, E. R., Bruce, P. J., and Santer, M. J., “Adaptive Shock Control Bumps," 52nd Aerospace Sciences Meeting, American Institute of Aeronautics and Astronautics, 2014. doi:10.2514/6.2014-0945.

[9] Nuebler, K., Lutz, T., Kraemer, E., Colliss, S., and Babinsky, H., "Shock Control Bump Robustness Enhancement," 50th AIAA Aerospace Sciences Meeting including the New Horizons Forum and Aerospace Exposition, American Institute of Aeronautics and Astronautics, 2012. doi:10.2514/6.2012-46. 
[10] Bruce, P. J. K., and Colliss, S. P., "Review of research into shock control bumps," Shock Waves, Vol. 25, No. 5, 2014, pp. 451-471. doi:10.1007/s00193-014-0533-4.

[11] Werner, M., "Application of an Adaptive Shock Control Bump for Drag Reduction on a Variable Camber NLF Wing," 2018 AIAA Aerospace Sciences Meeting, American Institute of Aeronautics and Astronautics, 2018. doi:10.2514/6.2018-0789.

[12] Schulz, V., and Schillings, C., "Optimal Aerodynamic Design under Uncertainty," Notes on Numerical Fluid Mechanics and Multidisciplinary Design, Springer Berlin Heidelberg, 2013, pp. 297-338. doi:10.1007/978-3-642-36185-2_13.

[13] Duvigneau, R., “Aerodynamic Shape Optimization with Uncertain Operating Conditions using Metamodels," resreport RR-6143, INRIA, 2007.

[14] Jones, D. R., Schonlau, M., and Welch, W. J., "Efficient Global Optimization of Expensive Black-Box Functions," Journal of Global Optimization, Vol. 13, No. 4, 1998, pp. 455-492. doi:10.1023/a:1008306431147.

[15] Maruyama, D., Liu, D., and Goertz, S., "An Efficient Aerodynamic Shape Optimization Framework for Robust Design of Airfoils Using Surrogate Models," Proceedings of the VII European Congress on Computational Methods in Applied Sciences and Engineering (ECCOMAS Congress 2016), Institute of Structural Analysis and Antiseismic Research School of Civil Engineering National Technical University of Athens (NTUA) Greece, 2016. doi:10.7712/100016.2450.8838.

[16] Li, J., Gao, Z., Huang, J., and Zhao, K., "Robust design of NLF airfoils," Chinese Journal of Aeronautics, Vol. 26, No. 2, 2013, pp. 309-318. doi:10.1016/j.cja.2013.02.007.

[17] Mazaheri, K., and Nejati, A., "The Multi-point Optimization of Shock Control Bump with Constant-Lift Constraint Enhanced with Suction and Blowing for a Supercritical Airfoil," Flow, Turbulence and Combustion, Vol. 96, No. 3, 2015 , pp. $639-666$. doi:10.1007/s10494-015-9671-8.

[18] Tian, Y., Liu, P., and Feng, P., "Shock control bump parametric research on supercritical airfoil," Science China Technological Sciences, Vol. 54, No. 11, 2011, pp. 2935-2944. doi:10.1007/s11431-011-4582-y.

[19] Gerhold, T., "Overview of the Hybrid RANS Code TAU," MEGAFLOW - Numerical Flow Simulation for Aircraft Design, Springer Berlin Heidelberg, 2015, pp. 81-92. doi:10.1007/3-540-32382-1_5.

[20] Gerhold, T., and Neumann, J., "The Parallel Mesh Deformation of the DLR TAU-Code,” Notes on Numerical Fluid Mechanics and Multidisciplinary Design (NNFM), Springer Berlin Heidelberg, 2006, pp. 162-169. doi:10.1007/978-3-540-74460-3_20.

[21] Meinel, M., and Einarsson, G., "The FlowSimulator framework for massively parallel CFD applications,” PARA $2010,2010$.

[22] Forrester, A. I., and Keane, A. J., "Recent advances in surrogate-based optimization," Progress in Aerospace Sciences, Vol. 45, No. 1-3, 2009, pp. 50-79. doi:10.1016/j.paerosci.2008.11.001.

[23] Han, Z.-H., Goertz, S., and Zimmermann, R., "Improving variable-fidelity surrogate modeling via gradient-enhanced kriging and a generalized hybrid bridge function," Aerospace Science and Technology, Vol. 25, No. 1, 2013, pp. 177-189. doi:10.1016/j.ast.2012.01.006.

[24] McKay, M. D., Beckman, R. J., and Conover, W. J., "Comparison of Three Methods for Selecting Values of Input Variables in the Analysis of Output from a Computer Code," Technometrics, Vol. 21, No. 2, 1979, pp. 239-245. doi: 10.1080/00401706.1979.10489755.

[25] Caflisch, R. E., "Monte Carlo and quasi-Monte Carlo methods," Acta Numerica, Vol. 7, 1998, p. 1. doi:10.1017/ s0962492900002804.

[26] Kucherenko, S., Albrecht, D., and Saltelli, A., "Exploring multi-dimensional spaces: a Comparison of Latin Hypercube and Quasi Monte Carlo Sampling Techniques," ArXiv e-prints, 2015.

[27] Sobol, I., "On the distribution of points in a cube and the approximate evaluation of integrals," USSR Computational Mathematics and Mathematical Physics, Vol. 7, No. 4, 1967, pp. 86-112. doi:10.1016/0041-5553(67)90144-9.

[28] Loeppky, J. L., Sacks, J., and Welch, W. J., "Choosing the Sample Size of a Computer Experiment: A Practical Guide," Technometrics, Vol. 51, No. 4, 2009, pp. 366-376. doi:10.1198/tech.2009.08040.

[29] Han, Z.-H., Abu-Zurayk, M., Goertz, S., and Ilic, C., "Surrogate-Based Aerodynamic Shape Optimization of a Wing-Body Transport Aircraft Configuration," Notes on Numerical Fluid Mechanics and Multidisciplinary Design, Springer International Publishing, 2018, pp. 257-282. doi:10.1007/978-3-319-72020-3_16. 
[30] Forrester, A. I. J., Sabester, A., and Keane, A. J., Engineering Design via Surrogate Modelling, John Wiley \& Sons, Ltd, 2008. doi:10.1002/9780470770801.

[31] Zimmermann, R., "Asymptotic Behavior of the Likelihood Function of Covariance Matrices of Spatial Gaussian Processes," Journal of Applied Mathematics, Vol. 2010, 2010, pp. 1-17. doi:10.1155/2010/494070.

[32] Storn, R., and Price, K., "Differential Evolution - A Simple and Efficient Heuristic for global Optimization over Continuous Spaces," Journal of Global Optimization, Vol. 11, No. 4, 1997, pp. 341-359. doi:10.1023/a:1008202821328.

[33] Alexandrov, N. M., Dennis, J. E., Lewis, R. M., and Torczon, V., "A trust-region framework for managing the use of approximation models in optimization,” Structural Optimization, Vol. 15, No. 1, 1998, pp. 16-23. doi:10.1007/bf01197433.

[34] Liu, D., and Goertz, S., "Efficient Quantification of Aerodynamic Uncertainty due to Random Geometry Perturbations," Notes on Numerical Fluid Mechanics and Multidisciplinary Design, Springer International Publishing, 2014 , pp. 65-73. doi:10.1007/978-3-319-03158-3_7.

[35] Kawai, S., and Shimoyama, K., "Kriging-model-based uncertainty quantification in computational fluid dynamics," 32nd AIAA Applied Aerodynamics Conference, American Institute of Aeronautics and Astronautics, 2014. doi:10.2514/6.2014-2737.

[36] Quagliarella, D., Petrone, G., and Iaccarino, G., "Optimization Under Uncertainty Using the Generalized Inverse Distribution Function,” Computational Methods in Applied Sciences, Springer Netherlands, 2014, pp. 171-190. doi:10.1007/978-94-0179054-3_10.

[37] van der Vaart, A. W., Asymptotic Statistics, CAMBRIDGE UNIV PR, 2011. URL https://www. ebook.de/de/product/ 3338604/a_w_van_der_vaart_asymptotic_statistics.html

[38] Dudley, R. M., Uniform Central Limit Theorems, Cambridge University Press, 1999. doi:10.1017/cbo9780511665622.

[39] Echard, B., Gayton, N., and Lemaire, M., "AK-MCS: An active learning reliability method combining Kriging and Monte Carlo Simulation,” Structural Safety, Vol. 33, No. 2, 2011, pp. 145-154. doi:10.1016/j.strusafe.2011.01.002.

[40] Bect, J., Ginsbourger, D., Li, L., Picheny, V., and Vazquez, E., "Sequential design of computer experiments for the estimation of a probability of failure," Statistics and Computing, Vol. 22, No. 3, 2011, pp. 773-793. doi:10.1007/s11222-011-9241-4.

[41] Schöbi, R., "Surrogate models for uncertainty quantification in the context of imprecise probability modelling," Ph.D. thesis, ETH Zürich, Zürich, Switzerland, 2017.

[42] Rowan, T. H., "Functional Stability Analysis of Numerical Algorithms," Ph.D. thesis, University of Texas Austin, Austin, TX, USA, 1990. UMI Order No. GAX90-31702. 